\title{
A characterization of boolean spaces
}

\section{C.E. Dickerson and M.E. Moore}

A boolean space is a compact Hausdorff space which is zerodimensional. In this paper, a boolean space $X$ is characterized in terms of its ring of real-valued functions $C(X)$. The result is sharpened for the case when $X$ is an $F$-space (every finitely generated ideal of $C(X)$ is principal).

\section{Introduction}

A boolean space is a compact Hausdorff space which is zerodimensionel. The purpose of this paper is to characterize a boolean space $X$ in terms of its ring of real-valued continuous functions $C(X)$. The result will be sharpened for the case when $X$ is an F-space (every finitely generated ideal of $C(X)$ is principal).

\section{B-rings}

Let $S$ be a commutative ring with identity 1 , and let $\left\{M_{\alpha} \mid \alpha \in A\right\}$ be the set of all maximal ideals of $S$. The Jacobson radical of $S$ is the set $J(S)=\cap\left\{M_{\alpha} \mid \alpha \in A\right\} . S$ is called a $B$-ring if for each integer $n \geq 3$ and each $s_{1}, \ldots, s_{n} \in S$ such that $\left(s_{1}, \ldots, s_{n-2}\right) \notin J(S)$ and $1 \in\left(s_{1}, \ldots, s_{n}\right)$, there exists $t \in S$ such that $1 \in\left(s_{1}, \ldots, s_{n-2}, s_{n-1}+t s_{n}\right) ;$ see [4] for details. Here, the notation $\left(s_{1}, \ldots, s_{n}\right)$ means the ideal of $s$ generated by $s_{1}, \ldots, s_{n}$.

Since every set of the form $M_{x}=\{f \in C(X) \mid f(x)=0\}$ is a maximal ideal of $C(X)$, it follows that if $g \in J(C(X))$ then $g \in M_{x}$ for each

Received 29 October 1974. 
$x \in X$ so that $g(x)=0$ for each $x \in X$, or equivalently, $g=0$. Hence, $J(C(X))=(0)$. We can now simplify the definition of $B$-rings in the special case of $C(X)$.

PROPOSITION 2.1. $C(X)$ is a B-ring if and only if $f, g, h \in C(X)$ with $f \neq 0$ and $I \in(f, g, h)$ implies there exists $t \in C(X)$ such that $I \in(f, g+t h)$.

Proof. The direct implication is obvious. To see the converse let $n \geq 3$ with $\left(f_{1}, \ldots, f_{n-2}\right) \notin(0)$ and $1 \in\left(f_{1}, \ldots, f_{n}\right)$; then $f_{1}^{2}+\ldots+f_{n-2}^{2} \neq 0$ and $z\left(f_{1}^{2}+\ldots+f_{n}^{2}\right)=\emptyset$, where $z\left(f_{1}^{2}+\ldots+f_{n}^{2}\right)$ denotes the zero set of the function $f_{1}^{2}+\ldots+f_{n}^{2}$. Note that $z\left(\left[f_{1}^{2}+\ldots+f_{n-2}^{2}\right]^{2}+f_{n-1}^{2}+f_{n}^{2}\right)=\emptyset$ must also hold. Consequently, $I \in\left(f_{1}^{2}+\ldots+f_{n-2}^{2}, f_{n-1}, f_{n}\right)$. By hypothesis, there exists $t \in C(X)$ such that $I \in\left(f_{1}^{2}+\ldots+f_{n-2}^{2}, f_{n-1}+t f_{n}\right)$. From this we see that $2\left(f_{1}^{2}+\ldots+f_{n-2}^{2}+\left[f_{n-1}+t f_{n}\right]^{2}\right)=2\left(\left[f_{1}^{2}+\ldots+f_{n-2}^{2}\right]^{2}+\left[f_{n-1}+t f_{n}\right]^{2}\right)=\emptyset$. Therefore, $1 \in\left(f_{1}, \ldots, f_{n-2}, f_{n-1}+t f_{n}\right)$.

\section{B-rings and boolean spaces}

In this section we shall assume that $X$ is a compact Hausdorf $f$ space. We begin by proving a lemma similar to Lemma 4.3 of [1].

LEMMA 3.1. Let $f, g, h \in C(X)$ and denote $g^{-1}(0, \infty)$ as $P(g)$ and $g^{-1}(-\infty, 0)$ as $N(g)$. If there is a connected subset $Z$ of $Z(f)$ such that $(Z \cap Z(h)) \cap P(g) \neq \varnothing$ and $(Z \cap Z(h)) \cap N(g) \neq \varnothing$, then for each $t \in C(X), \quad 1 \notin\left(f, g^{+t h}\right)$.

Proof. Note that there must be $x, y \in Z$ such that $(g+t h)(x)>0$ and $(g+t h)(y)<0$. Since $z$ is connected, the continuity of $g+t h$ implies the existence of some $z \in Z$ such that $(g+t h)(z)=0$. This shows that $Z(f) \cap Z(g+t h) \neq \emptyset$, or equivalently, $1 \notin(f, g+t h)$.

LEMMA 3.2. If $C(X)$ is a B-ring, then for each closed connected set $Z$ and each closed set $S, Z \cap S$ must be connected. 
Proof. The proof follows Lemma 4.5 of [1]. Suppose that $z$ is $a$ closed connected set and that $S$ is a closed set such that $2 \cap S$ is not connected. Write $Z \cap S=F_{1} \cup F_{2}$ where $F_{1}, F_{2}$ are disjoint non-empty closed subsets of $Z \cap S$, hence closed subsets of $X$. Since $X$ is assumed to be a compact Hausdorff space, and therefore normal, there are open sets $U_{1} \supseteq F_{1}$ and $U_{2} \supseteq F_{2}$ whose closures are disjoint. Put $U=U_{1} \cup U_{2}$. The closed sets $Z-U$ and $S-U$ are disjoint, hence contained in disjoint open sets $V_{1}, V_{2}$ respectively. By Urysohn's Lemma, choose $f, g, h \in C(X)$ such that $f(Z)=0$ and $f\left(X-V_{1}-U\right)=1$, $g\left(\bar{U}_{1}\right)=1$ and $g\left(\bar{U}_{2}\right)=-1, h(S)=0$ and $h\left(X-V_{2}-U\right)=1$. Then $f, g, h$ satisfy the hypothesis of the previous lemma and $l \in(f, g, h)$. It follows that $C(X)$ is not a $B$-ring.

THEOREM 3.3. Let $X$ be a compact Hausdorff space. If $C(X)$ is a $B$-ring, then $X$ is a boolean space.

Proof. Let $x \in X$. If $C$ is the connected component of $X$ containing $x$, then $C$ is a closed connected set. If $C \neq\{x\}$ then it would follow that the discrete set $C \cap\{x, y\}=\{x, y\}$ must be connected, where $y \in C-\{x\}$. We conclude that $C=\{x\}$ and, hence, $X$ is totally disconnected. By compactness, $X$ is zero-dimensional.

Next we prove the converse of Theorem 3.3. We begin by defining $A(X)$ to be all those functions $f \in C(X)$ whose range is a finite set. In particular, $A(X)$ contains the constant functions. It is well known that for compact spaces $X$, we may apply the Stone-Weierstrass Theorem to conclude that $A(X)$ is dense in $C(X)$, under the topology of uniform convergence, if $X$ is zero-dimensional.

THEOREM 3.4. If $X$ is a boolean space, then $C(X)$ is a B-ring.

Proof. If $f, g, h \in C(X)$ with $I \in(f, g, h)$, then a straightforward computation shows that there exist $\delta, \varepsilon>0$ such that if $f^{\prime}, g^{\prime}, h^{\prime} \in C(X)$ with $\left|f-f^{\prime}\right|<\varepsilon,\left|g-g^{\prime}\right|<\varepsilon$, and $\left|h-h^{\prime}\right|<\varepsilon$ then $\left|f^{\prime}\right|+\left|g^{\prime}\right|+\left|h^{\prime}\right|>\delta$. Let $\xi=\min (\varepsilon, \delta / 3)$ and choose $f^{\prime}, g^{\prime}, h^{\prime} \in A(X)$ within $\xi$ of $f, g, h$ respectively. Note then that $\left|f^{\prime}\right|+\left|g^{\prime}\right|+\left|h^{\prime}\right|>\delta$.

Since functions in $A(X)$ have finite range, it follows that there 
exist functions $u, v, w \in A(X)$ satisfying $u f^{\prime}=\left|f^{\prime}\right|, v g^{\prime}=\left|g^{\prime}\right|$, $w h^{\prime}=\left|h^{\prime}\right|$, and $|u|=|v|=|w|=1$. Define $c \in A(X)$ by $c=1 /\left(\left|f^{\prime}\right|+\left|g^{\prime}\right|+\left|h^{\prime}\right|\right)<1 / \delta$. Choosing $p=u c, g=v c$, and $t=w / v$ gives $1=p f^{\prime}+q\left(g^{\prime}+t h^{\prime}\right)$. Thus, we have appropriately written the identity in the subring $A(X)$.

Now set $d_{1}=f^{\prime}-f, d_{2}=g^{\prime}-g$, and $d_{3}=h^{\prime}-h ;$ then $\left|d_{i}\right|<\xi$ for each $i$ and

$$
\begin{aligned}
1 & =p\left(f+d_{1}\right)+q\left[\left(g+d_{2}\right)+t\left(h+d_{3}\right)\right] \\
& \leq|p f+q(g+t h)|+\left|p d_{1}+q d_{2}+q t d_{3}\right| .
\end{aligned}
$$

Letting $s=\left|p d_{1}+q d_{2}+q t d_{3}\right|$ it follows that $1-s \leq|p f+q(g+t h)|$. By direct calculation,

$$
\begin{aligned}
s & \leq|p| \cdot\left|d_{1}\right|+|q| \cdot\left|d_{2}\right|+|q t| \cdot\left|d_{3}\right| \\
& <(1 / \delta) \cdot \xi+(1 / \delta) \cdot \xi+(1 / \delta) \cdot \xi \leq 1 .
\end{aligned}
$$

This gives that $0<1-8 \leq|p f+q(g+t h)|$ so that $p f+q(g+t h)$ is a unit in $C(X)$. Since $p f+q(g+t h) \in(f, g+t h)$, it follows that $1 \in(f, g+t h)$.

Thus, we have shown that a compact space $X$ is a boolean space if and only if $C(X)$ is a $B$-ring. It is interesting to note that we did not need $f \neq 0$.

By assuming $X$ is Lindelöf and using the Stone-Čech compactification of $X$, one can easily show that $X$ is zero-dimensional if and only if $C^{*}(X)$ is a $B$-ring.

\section{4. $S B$-rings and boolean $F$-spaces}

Let $S$ be a commutative ring with identity. $S$ is called an $S B-$ ring if for each $s, c, d, e \in S$ with $s \in(c, d, e)$ and $c k J(S)$, it follows that $\varepsilon \in(c, d+t e)$ for some $t \in S$; see [4] for details.

A topological space $X$ is called an F-space if every finitely generated ideal of $C(X)$ is principal. $X$ is called a T-space if $C(X)$ is an Hermite ring; and $X$ is called a U-space if for each $f \in C(X)$ there exists a unit $u \in C(X)$ such that $f=u|f|$. In [1] it is shown that every $U$-space is a $T$-space. 
THEOREM 4.1. Suppose $X$ is a compact F-space. Then $X$ is a boolean space if and only if $C(X)$ is an SB-ring.

Proof. Since every $S B$-ring is a B-ring [4, p. 457], it suffices to show that if $X$ is a boolean $F$-space then $C(X)$ is an $S B$-ring. Now, every boolean $F$-space is a U-space [1, Theorem 5.5]. Hence, $X$ is a $T$-space and $C(X)$ is a Hermite ring. Since Hermite $B$-rings are $S B$-rings [4, Theorem 3.3], it follows that $C(X)$ is an $S B$-ring.

\section{References}

[1] Leonard Gillman and Melvin Henriksen, "Rings of continuous functions in which every finitely generated ideal is principal", Trons. Amer: Math. Soc. 82 (1956), 366-391.

[2] Leonard Gillman and Meyer Jerison, Rings of continuous fronctions (Ven Nostrand, Princeton, New Jersey; Toronto; London; New York; 1960).

[3] Paul R. Halmos, Lectures on Boolean algebras (Van Nostrand, Princeton, New Jersey; Toronto; New York; London; 1963).

[4] Marion Moore and Arthur Steger, "Some results on completability in commutative rings", Pacific J. Math. 37 (1971), 453-460.

[5] R.S. Pierce, Compact zero-dimensional metric spaces of finite type (Mem. Amer. Math. Soc., 130. Amer. Math. Soc., Providence, Rhode Island, 1972).

[6] Roman Sikorski, Boolean algebras, 2nd ed. (Frgebnisse der Mathematik und ihrer Grenzgebiete, Neue Folge, 25. Springer-Verlos, Berlin, New York; Academic Press, New York; 1964). 